\title{
Influence of measuring force on CMM accuracy
}

\author{
WANG Bin \\ Research Institute of Physical and Chemical Engineering of Nuclear Industry, Tianjin, China
}

\begin{abstract}
The measuring accuracy of CMM (Coordinate Measuring Machine) is influenced by many factors, such as temperature, humidity, measuring force and method of signal acquisition. For thin parts, the influence of measuring force is especially obvious. In this paper, the relationship between measuring force and measurement accuracy is studied for a thin part with a U-shaped cross-section. By analyzing the structure of the probe and establishing the force model, the influencing factors of the accuracy of CMM are obtained, and the influence of the contact deformation and the bending deformation on the measurement accuracy is analyzed from the point of view of material mechanics. At the same time, the measurement accuracy of different measuring cross-sections is analyzed. Through the research of this paper, the relationship between measuring force and CMM is established, and an effective method to improve the accuracy of CMM is also found.
\end{abstract}

\section{Structural analysis of trigger probe and error principle}

The probe is an important part of CMM, consisting of a measuring rod and a ruby head, which is mainly used to measure the working surface, move through the mechanical position of the measuring end, trigger the signal and collect a measurement data. The internal structure of the RDS XXT (Carl Zeiss' products) probe is shown in Fig. 1, the rod is fitted on the ring and the outer wall of the ring has a uniform contact terminals. When the rod is not measured, the outer wall of the ring is kept in contact with the terminals. One contact detachment is caused at least when the spherical end of the measuring rod is contacted with the workpiece, thus causing the circuit to disconnect, produce step signal, through the sampling circuit, will be along three axes direction of the coordinate data storage processing. Fig. 1-measuring rod, 2-ring cage.

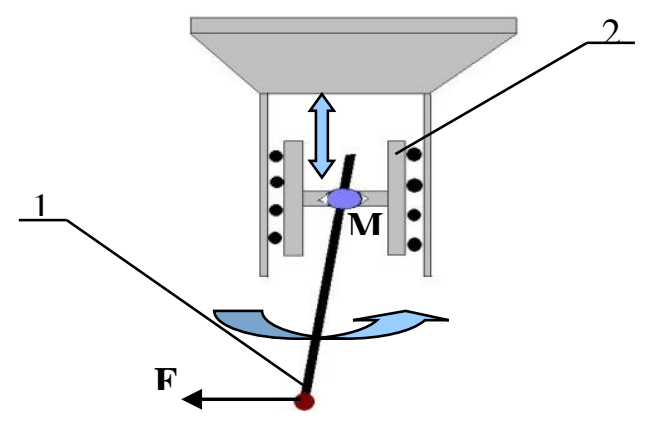

Fig. 1 Schematic diagram of the head probe

The process between the probe end and the measured part contacting to the probe trigger is "Pre-range" during the CMM is working. The Pre-range is not an error source, because a standard sphere with known radius is used to determine a reasonable probe radius to eliminate the effect of the average Pre-range during the probe certification process. The different triggering forces, that is the production of the Pre-range changes, as shown in Figure 2, the figure can be seen visible three convex angle, reflecting the amount of the change in the Prerange " $\delta$ ". This mode shows symmetry of the probe, which greatly reduces the ability of CMM to perform high-precision measurement.

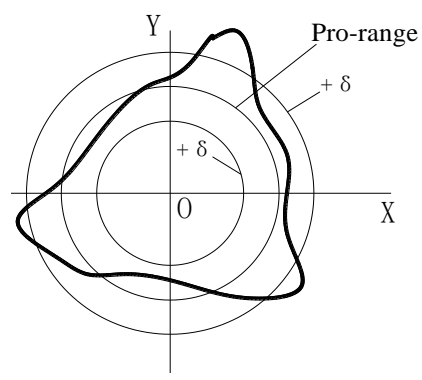

Fig. 2 The sketch of the convex angle of probe

\section{Stress analysis of the probe model}

The probe system of CMM is to issue the trigger pulse when the probe ball and workpiece collide, to send out the sampling signal to CMM, and the three coordinate values of the probe are stored at this time to determine its space position. The space coordinate equation can be obtained by more than three contact measurements on a plane. The center coordinate and radius can be obtained by touching the plane circle more than three times, and the shape of any complex space object may be determined ${ }^{[1]}$. 


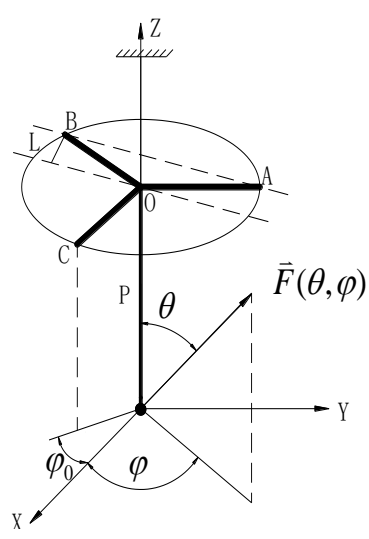

Fig. 3 Force model of dynamic trigger probe

As shown in Figure 3, the probe is positioned in the Cartesian coordinate system, and the probe is coincident with the $\mathrm{Z}$-axis. The probe length is $\mathrm{P}$, the projection of the cylindrical and the $\mathrm{X}$-axis angle are $\varphi_{0}$, the Force $F$ $(\theta, \varphi)$ is triggered by the end and the component surface. The direction of force $\mathrm{F}$ is determined by the polar angle $\theta$ and the azimuth $\varphi$. assuming that the direction of the probe is in line with the normal direction of the part surface, the probe is moved along the direction of the force.

$$
\vec{n}=\vec{F} / F=\vec{i} \sin \theta \cos \varphi+\vec{j} \sin \theta \sin \varphi+\vec{k} \cos \theta
$$

If the polar angle " $\theta$ " and azimuth " $\varphi$ " are in a suitable range, " $F$ " will cause the probe to rotate around the $A-B$ axis, and the signal will trigger when the cylinder $C$ leaves the contact terminals. According to the different F-direction of the force, the other two possible axes of rotation are $B-C$ and $A-C$, and the corresponding angle is $\varphi_{0} \pm 120^{\circ}$. In addition to rotation, Force " $F$ " also causes the probe to bend, resulting in bending displacemen.

\subsection{Contact deformation}

In order to ensure the reliable contact between CMM and the workpiece surface, it is necessary to impose a certain force acting on the workpiece, but the force will cause contact deformation of the workpiece. The size of contact deformation is related to the material and measuring force of the workpiece.

$$
\delta_{\text {contact }}=1.231\left((F / E)^{2}\left(R_{1}+R_{2}\right) / R_{1} R_{2}\right)^{1 / 2}
$$

" $F$ " is the measuring force, " $E$ " is the elastic modulus of the workpiece, the " $R_{1}, R_{2}$ " are the workpiece and the radius of the probe ball.

\subsection{Bending deformation}

The measuring force can also cause the bending deformation, and the bending deformation is mainly affected by the measuring position and the measuring force value. According to the knowledge of material mechanics, the bending deformation of workpiece under the action of measuring force is:

$$
\delta_{\text {bending }}=\frac{F L^{3}}{3 E I}
$$

" $F$ " is measuring force, " $E$ " is the elastic modulus of workpiece, " $I$ " is the workpiece section inertia moment, " $L$ " is the length of probe.

\section{Modal analysis of force measurement}

There are two kinds of CMM probes, one is the dynamic probe, the other is the static probe. The RDS XXT probe system is a dual-mode signal acquisition system which can realize dynamic (scanning) and static (triggering) measurement, under which the influence factors of the measuring force are analyzed respectively.

\subsection{Dynamic measurement mode}

It is possible for the probe to leave the surface of the workpiece and cause the measurement error. Because of the relative movement of the probe and the workpiece, when the relative motion speed is higher and the measuring force is small. In order to analyze the dynamic measuring force, the probe and the rod are simplified into the rod, which mass is " $m$ ", length is " $L$ ", and the static measuring force on the workpiece is " $F_{0}$ ". When measuring the probe relative to the workpiece surface, the measuring rod element revolves around the fulcrum, and the pendulum angle is " $\theta$ ", the dynamic equation of the measuring rod element is:

$$
J \ddot{\theta}=-\frac{M_{g l}}{2} \sin \theta+\left(F-F_{0}\right) L
$$

Because the measuring rod pendulum angle is very small, " $\theta$ "can be approximated as:

$$
\theta=\frac{h_{m}}{L} \sin \omega t
$$

the influence of the gravity moment of the measuring rod element can be ignored, the formula (4) can be reduced:

$$
F=F_{0}-\frac{M}{2} h_{m} \omega^{2} \sin \omega t
$$

In order to ensure reliable contact during measurement, the force value is $f \geq 0$, calculation:

$$
\omega_{\max }=\left(2 F_{0} / M h_{m}\right)^{1 / 2}
$$

Therefore, the workpiece is related to the maximum relative rotational speed and the static measuring force of the measuring ball during the scanning process. The probe and workpiece are separated when workpiece speed is greater than " $\Omega_{\max }$ ".

Therefore, in order to maintain good contact between the probe and the workpiece and to prevent the probe ball from being detached from the workpiece with lower 
rotational speed, which is equivalent to the static measurement, so that the influence of the measuring force change on the measurement result can be neglected [2]

\subsection{Static measurement mode}

The three-coordinate probe should maintain a constant measuring force in its contact with the static mode. The measuring force is mainly able to overcome the dust and oil film on the surface of workpiece, so as to ensure the stability of the measured value. However, in ensuring its stability, it should also control the value of the measuring force, to avoid deformation and scratches on the workpiece surface due to the excessive force. In the process of measuring the workpiece, there are two kinds of errors in the measurement result of geometrical dimension, one is the contact deformation of the surface, the other is the error due to the longitudinal deformation and bending ${ }^{[3]}$.

When two objects are in contact with each other, a certain deformation is produced by squeezing. At the same time, because of the influence of the stress on the value of the deformation, the Gelz theory is used to analyze and calculate the deformation of the stress relation and stress effect. The spherical probe is the most commonly used in the contact measuring method, in which the contact deformation affects the measurement precision of the geometrical dimension, in which case, the effect of measuring force on the measurement result can be reduced by increasing the radius of the sphere.
According to the above analysis, the main influencing factors in the static measurement process are contact deformation and bending deformation. The RDS XXT probe system is configured with three triggering measuring force modes, which are sensitive trigger, standard trigger and strong trigger. The sensitive mode is mainly used to measure the smooth surface of the workpiece, such as plastic and glass. The standard mode is used for most metal materials. The strong mode is used for surface roughness testing of composite materials. Therefore, the standard trigger mode is more suitable for the detection of metal parts, and the relationship between the measuring force and length of the bar is shown in Fig. 4.

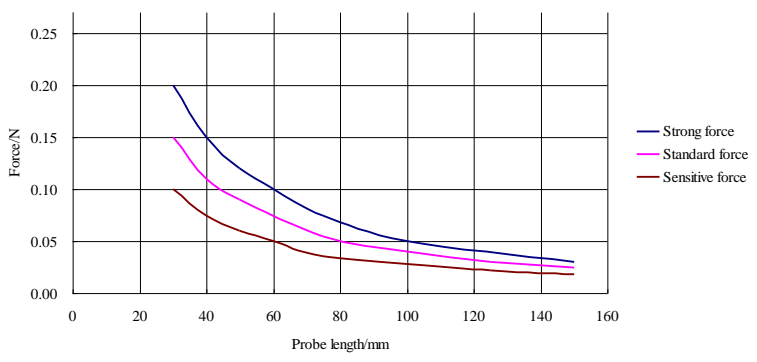

Fig. 4 Relationship between measuring force and probe length

\subsubsection{Calculation of contact deformation}

The probe ball radius is $1.5 \mathrm{~mm}$, substituting formula (2), and the deformation is shown in table 1 .

Table 1. Calculation value of measuring force and contact deformation

\begin{tabular}{ccc}
\hline Probe length/mm & Measuring force $/ \mathbf{m N}$ & Contact deformation $/ \boldsymbol{\mu m}$ \\
30 & 150 & 1.8 \\
40 & 110 & 1.5 \\
50 & 90 & 1.3 \\
75 & 55 & 0.9 \\
100 & 40 & 0.7 \\
125 & 30 & 0.6 \\
150 & 25 & 0.5 \\
\hline
\end{tabular}

The contact deformation of workpiece is only $1.8 \mu \mathrm{m}$, which corresponded to the minimum probe length. Because the CMM measurement error is $1.9 \mu \mathrm{m}$, so the deformation can be neglected.

\subsubsection{Calculation of bending deformation ${ }^{[4]}$}

The bending deformation of workpiece is not only related to the material of workpiece, but also has great influence on the fixed height. The structure of the Ushaped piece is shown in Figure 5. 


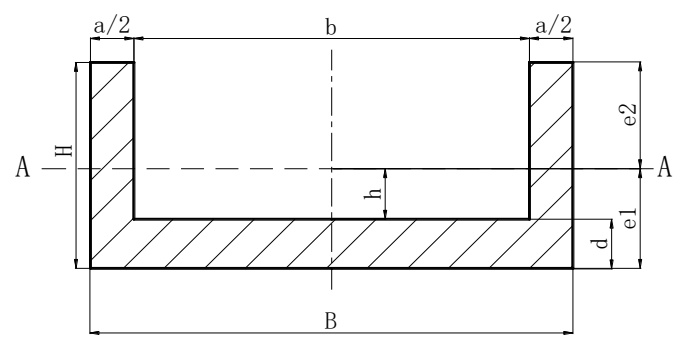

Fig. 5 Simplified model diagram of inertial moment
The inertia moment is calculated by the formula (8).

$$
I=\frac{B e_{1}^{3}-b h^{3}+a e_{2}^{3}}{3}
$$

The relationship between the bending deformation val ue and the different fixed height $\mathrm{H}$ can be calculated ${ }^{[5]}$. Height variation $\triangle H$ is $1 \mathrm{~mm}$. Substituting formula (3), $t$ he relationship between the measuring force and the bend ing deformation of the U-shaped parts in different measu ring sections can be calculated as shown in table $2^{[6]}$.

Table 2 .Calculation of measuring force and bending deformation

\begin{tabular}{ccccccc}
\hline & $\begin{array}{c}\text { Measuring force } \\
\text { FixN }\end{array}$ & $\mathbf{1 5 0}$ & $\mathbf{9 0}$ & $\mathbf{5 5}$ & $\mathbf{4 0}$ & $\mathbf{2 5}$ \\
$\mathbf{\Delta \mathbf { H } / \mathbf { m m }}$ & $\mathbf{2}$ & 0.5 & 0.6 & 0.8 & 0.9 & 1.2 \\
& $\mathbf{4}$ & 0.6 & 0.9 & 1.1 & 1.4 & 1.7 \\
& $\mathbf{6}$ & 1.2 & 1.3 & 1.6 & 1.9 & 2.2 \\
& $\mathbf{8}$ & 2.2 & 2.4 & 2.5 & 2.8 & 3.1 \\
& $\mathbf{1 0}$ & 4.0 & 4.2 & 4.5 & 5.2 & 6.2 \\
\hline
\end{tabular}

\section{Conclusion}

With the increase of measuring force, the bending deformation is also increasing. And with the increase of the fixed height, the bending deformation reaches $6.2 \mu \mathrm{m}$, so the influence of bending deformation is the key factor of the measurement error.

By analyzing the principle of three coordinate measuring machine and the deformation mechanism caused by measuring force, it is shown that the influence of the U-shaped parts is mainly reflected in the bending deformation. The instability of the measured results caused by deformation can be improved by reducing the force and determining the reasonable measuring section.

\section{References}

1. Wang Yunqing, Li Qingxiang, Zhou Chiying. Analysis of stylus measurement force in contact
2. Contour Measurement [j]. Modern metrology Test, 1996, (1); 18-21.

3. De Guangyun, Jianhua, Li Ping. Dynamic performance analysis of contact micrometer for probe [j]. Xi ' an Industrial college journal, 2004, 24 (1): 11-14

4. Statham A. Coordinate measurement Centre for Metrology, The University of Birmingham, Birmingham, 1991

5. Hou Yu, Lu Jin, CMM theory and method of shape error evaluation $[\mathrm{J}]$, Journal of Instrumentation, 1996, 17(6): 618-623

6. Fei Y. T. et al., Symmetrizable connection and combined calibration method for accuracy measurement of CMM. Measurement Tech. And Intelligent Instruments. 1993; 1462-1464 